\title{
RESINA DE RESISTÊNCIA A UMIDADE PARA PAPEL E PAPEL CARTÃO EM CONTATO COM ALIMENTOS COM BAIXOS NÍVEIS DE EPICLORIDRINA E SEUS SUBPRODUTOS.
}

\author{
Roberta de O. Motta $^{1^{*}}$, Koiti Araki ${ }^{*}$ \\ 1 - Instituto de Química, Universidade de São Paulo (USP), São Paulo, SP \\ rolivmotta@hotmail.com
}

\begin{abstract}
Resumo: As atualizações nas regulamentações internacionais e nacionais direcionaram as indústrias de papel para contato com alimentos a desenvolverem produtos que minimizem os perigos a saúde e ao meio ambiente. Assim, segundo as novas normativas, os agentes de resistência à umidade à base de resina poliamidoamina-epicloridrina (PAE) aplicados em embalagens para contato com alimentos devem ter seus níveis residuais de epicloridrina e de seus subprodutos de reação rigorosamente controlados, tornando os processos tradicionais de fabricação baseados em reações de poliamidoamina com excesso de epicloridrina inadequados. Neste trabalho descrevemos a utilização de um agente finalizador de cadeia na reação de formação da poliamidoamina e posterior ajuste da relação estequiométrica da poliamidoamina e a epicloridrina para controlar os residuais e subprodutos de hidrólise, assim atendendo aos rigorosos padrões exigidos nas novas regulamentações.

Palavras-chave: papel para contato com alimentos, regulamentações, poliamidoamina-epicloridrina (PAE), epicloridrina e subprodutos.
\end{abstract}

\section{WET STRENGTH RESIN FOR PAPER AND PAPERBOARD FOOD CONTACT WITH LOW CONTENT OF EPICHLOROHYDRIN RESIDUAL AND BYPRODUCTS}

\begin{abstract}
Updates to international and national regulations have led the food contact paper industries to develop products that minimize health and environmental hazards. Thus, according to the new regulations, moisture resistance agents based on polyamidoamine-epichlorohydrin resin (PAE) applied in packaging must have their residual levels of epichlorohydrin and its reaction byproducts more strictly controlled, making inappropriate traditional manufacturing processes based on polyamidoamine reactions with excess epichlorohydrin. In this work, we describe the use of a chain-finishing agent in the formation reaction of polyamidoamine followed by subsequent adjustment of the stoichiometric relationship of polyamidoamine and epichlorohydrin to control the residuals and hydrolysis byproducts, thus meeting the strict standards of the new regulations.
\end{abstract}

Keywords: food contact paper, regulations, polyamidoamine-epichlorohydrin (PAE), epichlorohydrin and byproducts.

\section{Introdução}

As mais recentes atualizações nas legislações internacionais e nacionais estão direcionando as indústrias a desenvolverem produtos que não ofereçam perigos a saúde e ao meio ambiente. $\mathrm{Na}$ indústria de papel, principalmente no segmento de papel para contato com alimentos, um controle rigoroso é realizado por órgãos regulamentadores como ANVISA, FDA e BfR de todos os insumos utilizados no processo de fabricação, trazendo um novo desafio para as indústrias químicas.

As resinas, utilizadas como agente de resistência à umidade a base de poliamidoamina-epicloridrina (PAE) possuem altos níveis de epicloridrina e seus subprodutos de reação, os quais não mais atendem aos novos padrões impostos pelas normas. A necessidade de adequação, fez com que fosse iniciado a pesquisa de uma nova tecnologia que além de garantir a competitividade no segmento de papel e celulose atenda as legislações, minimizando o impacto ambiental dos efluentes e a exposição da população aos perigos para a saúde que estes produtos podem gerar.

O objetivo deste trabalho é desenvolver uma nova tecnologia em PAE, insumo para a fabricação de embalagens de papel para contato com alimentos com níveis reduzidos de compostos halogenados orgânicos adsorvíveis (AOX), subprodutos e resíduos da síntese da PAE como 1,3-dicloro-2- 
propanol, 3-cloro-1,2-propanodiol, epicloridrina e etilenoimina. (Figura 1), de modo que atenda às novas limitações expostas na legislação.

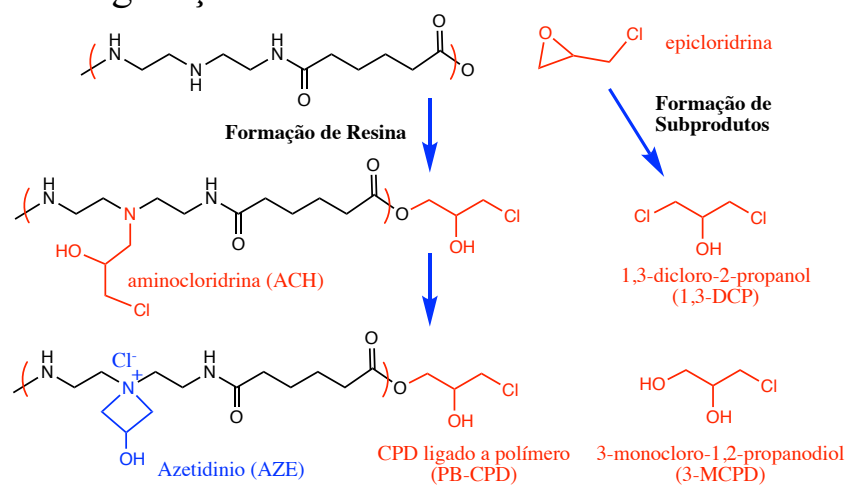

Figura 1 - Esquema mostrando a síntese da poliamidoamina-epicloridrina e seus subprodutos.

Algumas rotas vêm sendo estudadas e apresentadas em diferentes patentes e artigos como alternativas para redução da quantidade de produtos da hidrólise e da epicloridrina, e foram utilizadas como base para definir as direções que seguimos para alcançar o objetivo.

\section{Experimental}

Os processos para preparação das resinas de poliamidoamina-epicloridrina são bastante conhecidos e envolvem uma reação de poliamidoamina, em base aquosa, com um excesso de epicloridrina para converter completamente os grupos amina em adutos de poliamidoamina-epicloridrina. Reação de grupo amino de amina secundária com a epicloridrina com formação do intermediário $\mathrm{ACH}$, conforme esquema mostrado na Figura 1.

Após adicionar a epicloridrina e acompanhar a evolução da reação exotérmica, é iniciado o aquecimento para que ocorra a formação de ligações cruzadas e aumentar a viscosidade quando a temperatura da mistura reacional começa a diminuir. Nesta reação são gerados os íons azetidíneos responsáveis pela formação de uma forte rede de ligações cruzadas com a fibra de celulose conferindo resistência à umidade ao papel (Figura 2).

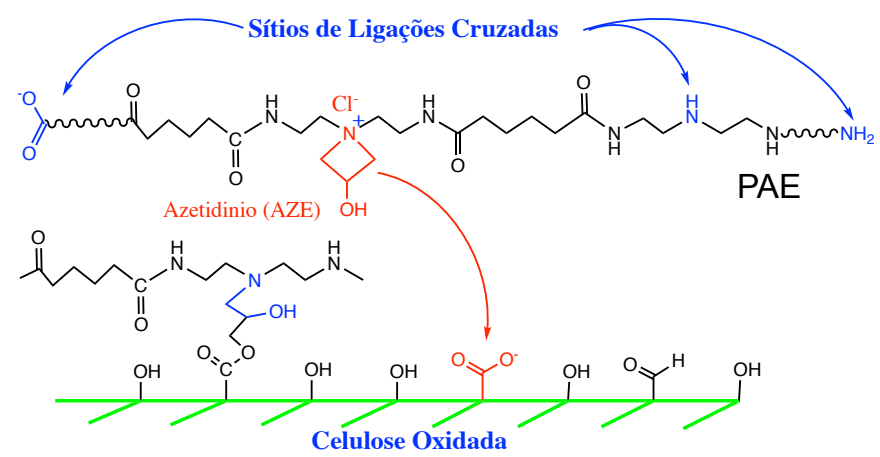

Figura 2 - Esquema mostrando a formação de uma rede de ligações cruzadas com a fibra de celulose pelo

PAE.

O problema foi atacado separando-o em duas etapas. Primeiramente a reação de formação da poliamidoamina foi otimizada, utilizando um agente finalizador de cadeia, a fim de bloquear seu 
crescimento descontrolado, e ajustando a estequiometria para que o polímero seja robusto. Foram realizados ensaios avaliando cada variável pré-determinada individualmente. A segunda etapa foi direcionada ao ajuste da reação de formação da poliamidoamina-epicloridrina onde foram otimizados parâmetros tais como a relação estequiométrica, temperatura e tempo de processo.

\section{Resultados e Discussões}

Os resultados apresentados a seguir se referem ao ensaio que apresentou o melhor desempenho em comparativo com o padrão atual de mercado, DYNAWET, o qual se tornou obsoleto para os fins aqui propostos, com as novas atualizações nas legislações. $O$ ensaio foi submetido a análises físicoquímicas de teor de não voláteis, $\mathrm{pH}$ e estabilidade. Posteriormente foram realizadas análise de aplicação e caracterização de 1,3-dicloro-2-propanol (1,3-DCP) e resíduo de epicloridrina por CGMS. (Tabela 1)

Tabela 1 - Resultados comparativos de desempenho DYNAWET x Ensaio.

\begin{tabular}{|l|l|l|}
\hline Características & \multicolumn{1}{|c|}{ DYNAWET } & \multicolumn{1}{c|}{ Ensaio } \\
\hline Teor de não voláteis & $20 \%$ & $20 \%$ \\
\hline pH & 3,03 & 3,38 \\
\hline Estabilidade T ambiente, $24 \mathrm{~h}$ & Estável & Estável \\
\hline Estabilidade $45^{\circ} \mathrm{C}-7$ dias & Estável & Estável \\
\hline \%DCP (ppm) & 660 & 38 \\
\hline \%Epicloridrina & $<10 \mathrm{ppm}^{*}$ & $<10 \mathrm{ppm} *$ \\
\hline Aplicação Celulose Branca** & $96 \%$ & $11 \%$ \\
\hline
\end{tabular}

*Limite de detecção.

**Equipamento dinamômetro.

Com base nos resultados pode-se observar que o produto reduziu o teor de 1,3-DCP em aproximadamente 20 vezes mantendo o produto estável em diferentes condições e com um desempenho na aplicação adequado aos padrões requisitados pelo mercado.

\section{Conclusões}

Um processo eficiente para preparação de uma resina poliamidoamina-epicloridrina com baixos teores residuais de epicloridrina e dos subprodutos de reação, atendendo aos rigorosos padrões exigidos pelas atuais normas nacionais e internacionais de papel para contato com alimentos foi desenvolvido, atingindo os objetivos propostos.

\section{Referências Bibliográficas}

1. WAN-YUN, L. Development and validation of an improved method for determination of chloropropanols in paperboard food packaging by GC-MS. New Jersey, 2012.

2. DULANY, A. M. Resins with reduced epichlorohydrin hydrolyzates. US Pat. 5,256,727, 26 out. 1993.

3. ANVISA, RDC No 88, DE 29 DE JUNHO DE 2016, Brasil.

4. MILLER, J. A. Process for the production of improved polyamidopolyamine epichlorohydrin resins. US Pat. 5,171,795, 15 dez. 1992.

5. GORZYNSKKI, M. Epihalohydrin-based resins having a reduced halogen content. US Pat. 5,516,885, 14 maio 1996. 
6. WILMINGTON, A. J. A., process of preparing polyamine-epihalohydrin resins with reduced byproduct content. EU Pat. 07000113.6, de 12 de junho de 2000.

7. OBOKATA, T.; ISOGAI, A. The mechanism of wet-strength development of cellulose sheets prepared with polyamidoamine-epichlorohydrin (PAE) resin. Colloids and Surfaces A: Physicochem. Eng. Aspects 302, p.525-531, 2007.

8. ALLEN, A. J., Silyl-linked polyamidoamine and their preparation. US Pat. 6,315,865, 13 November 2001.

9. BOWER, B. K., Wet strenght resins having reduced levels of organic halogen by-products. US005614597A. 25 de march 1997. 